Saudi Journal of Oral and Dental Research

Abbreviated Key Title: Saudi J Oral Dent Res

ISSN 2518-1300 (Print) |ISSN 2518-1297 (Online)

Scholars Middle East Publishers, Dubai, United Arab Emirates

Journal homepage: https://saudijournals.com

\title{
Working Length Determination Using Apex Locator and Cone Beam Computed Tomography in Presence of Three Irrigating Solutions in Primary Teeth: An in Vitro Comparative Study
}

\author{
Anindita Talukdar ${ }^{1 *}$, Prakash Chandra ${ }^{2}$, Latha Anandakrishna
}

${ }^{1}$ Private consultant, Pedodontist Guwahati, India

${ }^{2,3}$ Professor, Department of Paediatric Dentistry, Faculty of Dental Sciences, M S Ramaiah University of Applied Sciences, Bengaluru, India

DOI: $10.36348 /$ sjodr.2021.v06i02.002 $\quad$ | Received: 02.02.2021 | Accepted: 12.02 .2021 | Published: 16.02 .2021

*Corresponding author: Dr. Anindita Talukdar

\section{Abstract}

The purpose of the present study was to determine the working length using electronic apex locator in various intracanal mediums and cone beam computed tomography scan and to compare the lengths with the actual length of the tooth. Methods: In an in vitro setting, a total of 45 primary molar roots were decoronated till the cemento enamel junction for standardization, working length was measured with the Apex DSP (Septodont, France) at the 'apex' in presence of three different irrigating solutions namely $0.9 \%$ saline, $0.2 \%$ chlorhexidine and $2.5 \%$ sodium hypochlorite. The radiological working length was then calculated with cone beam computed tomography scans. The collected data was analyzed using descriptive statistics. Independent ${ }^{6} t$ ' test was done to compare the working length between the groups. Results: Findings showed there was significant difference in working length determination using apex locator in presence of saline and chlorhexidine when compared to sodium hypochlorite. Also working length determination with cone beam computed tomography compared to actual length did not show any significant difference. Conclusion: Apex DSP can be considered to be accurate across a variety of irrigants commonly used in the pediatric endodontic practice. A CBCT scan can be used as a diagnostic tool for pulpal treatment when treating multiple carious lesions.

Keywords: Working length, apex locator, chlorhexidine, sodium hypochlorite, Cone beam computed tomography.

Copyright () 2021 The Author(s): This is an open-access article distributed under the terms of the Creative Commons Attribution 4.0 International License (CC BY-NC 4.0) which permits unrestricted use, distribution, and reproduction in any medium for non-commercial use provided the original author and source are credited.

\section{INTRODUCTION}

Endodontic treatment in pedodontics requires treatment of pulpally involved primary and young permanent teeth in children to maintain the integrity and health of the oral tissues and also it is desirable to maintain pulp vitality wherever possible[1]. In permanent teeth, definitive rules for successful endodontic therapy have been established such as dense obturation and apical/coronal seal. In contrast, in pediatric endodontics, because of open apices, there are technical hitches in estimating the exact root canal length [2]. Working length determination is an important step in successful root canal therapy [3]. The accurate determination of working length has a major impact on the outcome of successful endodontic treatment in primary dentition. Frequently used method for determining working length are periapical radiographs. However there are various shortcomings in using conventional radiographs which includes the sensitivity of the technique, subjectivity and errors due to image magnification, distortions or the superimposition of anatomical structures [4].

Moreover, while performing endodontic procedure in children various factors should be taken into consideration like the time taken for the procedure, feasibility of taking multiple radiographs for working length determination. In such cases apex locator might be of importance as this device can be used with ease and also reduces the number of radiographs. Again with the advent of newer technologies like Cone-beam computed tomography (CBCT) which is a contemporary radiological imaging system, undistorted images can be obtained with a significantly lowereffective radiation dose than conventional computed tomography[5]. Hence the null hypothesis is that there is no significant difference in the diagnostic ability of CBCT scan and apex locator in different solutions when 
Anindita Talukdar et al; Saudi J Oral Dent Res, Feb, 2021; 6(2): 92-96

compared to actual length in determination of working length.

\section{MATERIALS \& METHODS}

An in vitro comparative study was conducted in the Department of Pedodontics and Preventive Dentistry at M.S. Ramaiah Dental College, Bengaluru. A total of 45 primary molar root canals extracted for therapeutic reasons from children visting the Outpatient Department were used for the study, consent were taken from the patients for using the extracted teeth. The inclusion criteria of teeth were primary molars without root resorption, teeth with irreversible pulpitis and recurrent chronic infection. Teeth that had calcified root canals, teeth with developmental anomaly or curvatures were excluded from the study.

\section{METHOD OF COLLECTION DATA}

Teeth extracted were collected and stored in $10 \%$ formalin solution until used. The teeth were sectioned horizontally with a diamond disc at the cemento enamel junction to provide unrestricted access to the canal and to obtain a flat surface [2]. A point was marked with a permanent marker to serve as reference for the placement of the rubber stop. In each multirooted tooth, one canal was randomly chosen for study. The teeth were numbered and embedded in an alginate model. Canals were then cleaned with $5 \mathrm{~mL}$ of saline solution and dried with paper points. Then, the patency of the canal was checked with a size $10 \mathrm{~K}$ Flexofile. After that the actual working length of each tooth was measured by introducing a number $10 \mathrm{k}$ file into the canal until its tip emerged through the apical foramen. A magnifying glass was used to visualize the tip of the file. Then the canal was irrigated using normal saline and the working length was determined using electronic apex locator followed by irrigation with water and drying the canal with paper points. Again introducing the other two irrigants namely $0.2 \%$ chlorhexidine and $2.5 \%$ sodium hypochlorite one after the other the working length was recorded after thorough water irrigation and drying the canals between each irrigants placement. Apex D.S.P. (Septodont, France) was used as the apex locator in presence of specific irrigant. Apex D.S.P is the most advanced apex locator of its kind based on D.S.P (Digital Signal Processing) technology; it offers superior accuracy and reliability. Utilizing sophisticated software, the Apex D.S.P immediately converts analog signals to digital format and transmits them via short cables. Other Apex Locators utilize analog signals that are transmitted over long cables, reducing the signal and increasing the exposure to distortion. Apex D.S.P delivers accurate, clearly displayed information everytime. All the measurements were calculated by a single examiner. Thereafter a cone beam computed tomography was made using machine CBCT CS 9300 Premium for each specimen. The CBCT scan was made using parameters $60 \mathrm{kvp} 3.2 \mathrm{~mA}$ and $8.01 \mathrm{sec}$ with voxel size of 300 microns. Then the scans were evaluated in both buccolingual and mesiodiatal direction the average of both the measurements was taken as the final working length. Both the apex locator and CBCT working length values was then compared with the actual length and evaluated for accuracy.

\section{STATISTICAL ANALYSIS}

Data was collected, compiled and analysed using SPSS statistics software, version 20.0 (SPSS Inc., Chicago, IL, USA). Descriptive statistics were analysed and presented in terms of mean and standard deviation. Independent ' $t$ ' test was used to compare the working lengths between the groups. The $p$ value $<0.05$ was considered as statistically significant.

\section{RESULTS}

A strongly significant positive correlation between between the first three groups that is actual length and CBCT, saline, chlorhexidine groups when compared to actual working length whereas a negative correlation was seen with sodium hypochlorite group. The table 3 shows that among all the groups CBCT scan determined the correct working length with $73.33 \%$ and EAL in presence of saline showed $51.11 \%$ correct estimation. However between saline and chlorhexidine saline showed better clinical significance with an overestimation of $13.33 \%$ compared to chlorhexidine which showed $22.22 \%$. To access the intra examiner reliability kappa test was done and the value was found to be 0.95 .

Table-1: Comparison of working length measured using different conditions/techniques in comparison with actual working length $(\mathrm{mm})$

\begin{tabular}{|l|c|c|c|c|}
\hline & Mean \pm SD & difference & t value & P value \\
\hline Actual WL $(\mathrm{mm})$ & $11.26 \pm 1.66$ & - & - & \\
\hline CBCT WL $(\mathrm{mm})$ & $11.31 \pm 1.50$ & -0.044 & -0.219 & 0.828 \\
\hline Apexlocator working length(mm) : Saline & $10.82 \pm 1.48$ & 0.444 & 2.881 & 0.006 \\
\hline Apexlocator working length(mm):Chlorhexidine & $11.15 \pm 1.79$ & 0.111 & 0.522 & 0.606 \\
\hline Working length (mm) : Sodium hypochlorite & $10.46 \pm 2.41$ & 0.80 & 1.70 & 0.096 \\
\hline
\end{tabular}


Anindita Talukdar et al; Saudi J Oral Dent Res, Feb, 2021; 6(2): 92-96

Table-2: Pearson Correlation

\begin{tabular}{|l|c|r|}
\hline \multicolumn{1}{|c|}{ Pearson Correlation } & r value & P value \\
\hline Actual WL (mm) vs CBCT WL (mm) & 0.631 & $<0.001^{* *}$ \\
\hline Actual WL (mm) vsApexlocator working length Saline & 0.788 & $<0.001^{* *}$ \\
\hline Actual WL (mm) vsApexlocator working length Chx & 0.667 & $<0.001^{* *}$ \\
\hline Actual WL (mm) vsWorking length $(\mathrm{mm}) \mathrm{NaoCl}$ & -0.182 & 0.232 \\
\hline
\end{tabular}

Table-3: Comparison of the overestimation and underestimation of working length

\begin{tabular}{|c|c|c|c|}
\hline & Correct estimation $\mathbf{( \% )}$ & $\begin{array}{c}\text { Over estimation } \\
(\mathbf{\%})\end{array}$ & $\begin{array}{c}\text { Under estimation } \\
(\mathbf{\%})\end{array}$ \\
\hline CBCT & 73.33 & 4.4 & 22.22 \\
\hline Saline & 51.11 & 13.33 & 35.55 \\
\hline Chlorhexidine & 48.82 & 22.22 & 31.11 \\
\hline Sodium hypochlorite & 6 & 26.66 & 66.66 \\
\hline
\end{tabular}

Table-4: The effective doses of various radiographic Procedures

\begin{tabular}{|l|l|}
\hline Radiographic procedure & Effective dose $(\mu \mathbf{S v})$ \\
\hline Intra-oral periapical & 5 \\
\hline Full mouth series & 35 \\
\hline Panoramic & $9-26$ \\
\hline i-CAT $($ CBCT) & $14.4-29.6^{*}$ \\
\hline
\end{tabular}

*After collimation and reducing the FOV to particular jaw. CBCT: Cone beam computed tomography, FOV: Field of view, i-CAT

\section{DISCUSSION}

Pulpectomy is one of the most common treatment options in primary teeth with infected pulp [6]. Although considered a safe procedure for primary teeth [7], various factors must be taken into consideration before starting treatment, long appointments may be exhausting for young patients and the results of diagnostic procedures which require a child's cooperation are less reliable compared with adult patients [8]. Establishment of correct working length is crucial in root canal treatment because sufficient data suggests that instrumentation either beyond or too short of apex can adversely affect the outcome of the treatment [9].

Over the years periapical radiographs have been used extensively for determining the working length, but radiation exposure concerns, time taking chemical processing and observers' bias in radiographic interpretation puts electronic methods of length determination in the front seat. Recent advances in the field of oral radiology are being channeled toward reducing exposure time and obtaining greater image definition [7]. Minor degrees of resorption may not be obvious radiographically and might lead to overestimation of working length ${ }^{8}$. Electric root length determination may be helpful to mask the disadvantages of radiographic examination in teeth with resorption [9]. Moreover the effect of canal condition on working length determination should also be considered as early generation electronic apex locators were often unreliable in the presence of conductive fluids. However, newer apex locators locates the foramen under any canal condition (wet, dry, pus, blood, irrigants etc.) as a result of its multi-frequency technology [10].

Previous studies done to evaluate efficacy of apex locator in presence of various irrigants reported that the canals filled with electroconductive solutions greatly reduce the impedance and there were tendencies toward short measurements in high electroconductive fluids like sodium hypochlorite, whereas longer measurements were in the lower electroconductive fluids like normal saline and chlorhexidine which is in agreement with our study $[11,12]$.

When comparing the efficacy of apex locator in presence of individual irrigants, the results of this study are in agreement with a similar study which reported that in presence of conducting fluids like sodium hypochlorite the accuracy of the apex locator decreased, with the greatest deviation from the actual length in the presence of sodium hypochlorite also the study concluded that chlorhexidine glucanoate showed the best results which is in agreement with our study [13]. When the saline and chlorhexidine group were compared working length in presence of saline showed a correct estimation of $51.11 \%$ whereas chlorhexidine showed $48.82 \%$ as shown in table 3.

The results of this study showed measurements of the APEX DSP (Septodont, France) were more precise in the presence of $0.9 \%$ saline and $0.2 \%$ chlorhexidine glucanoate. Presence of $\mathrm{NaOCl}$ solution in the canal almost inhibited its working ability, indicating that when the canals were filled with strong electrolytes, the results were negatively affected; giving measurements short of working length which is in 
Anindita Talukdar et al; Saudi J Oral Dent Res, Feb, 2021; 6(2): 92-96

agreement with another study done where measurements done with ProPex were more precise in the presence of chlorhexidine than sodium hypochlorite [10]. A strongly significant positive correlation was seen with saline and chlorhexidine groups when compared to actual working length whereas a negative correlation was seen with sodium hypochlorite group as seen in table 2.

Another important observation that was drawn from the study was the ability of the apex locator to produce accurate estimation and also the number of overestimation and underestimation of the working length. As in pediatric endodontics care should be taken not to extend the preparation beyond the working length overestimation of even $1 \mathrm{~mm}$ has detrimental effect on the succadenous tooth. Also optimal healing occurs when instrumentation and compact sealing are confined inside the root canal system [13]. In this study apex locator WL in presence of saline showed an overestimation of $13.33 \%$ whereas chlorhexidine showed $22.22 \%$ implying that saline has a more clinical significance than chlorhexidine.

The accuracy of the canal measurements with CBCT when compared to actual working length showed a Mean \pm SD of $11.31 \pm 1.50$ showed no significant difference compared to the actual working length with Mean \pm SD of $11.26 \pm 1.66$. The pearson correlation coefficient showed highly positive correlation of 0.631 with actual length. Also in the present study the percentage of accurate estimation of working length with CBCT was $73.3 \%$ which when compared to the apex locator in presence of saline group was only $51.11 \%$ which showed that CBCT could accurately measure the working length when compared to apex locator. However an in vitro study done on permanent molars using Raypex $6^{\circledR}$ reported that under the experimental conditions, the Raypex $6^{\circledR}$ was more accurate than CBCT scans for WL determination [14], which is not in agreement with this study where CBCT showed better accuracy as compared to Apex DSP(Septodont, France) which might be due to the morphological difference between deciduous and permanent teeth such as open apices in deciduous teeth [2] and also the ability to locate the apical foramen in permanent teeth precisely compared to deciduous teeth.

However within the limitations of the study as it was an in vitro study CBCT was possible however invivo $\mathrm{CBCT}$ for pulpectomy in children stills remains controversial because of the health effects of radiation. The most common indication for CBCT is malocclusion and orofacial anomalies in the primary and permanent dentition age groups, whereas the localisation of impacted teeth is the most common indication in the mixed dentition age group. Generally, CBCT was indicated in orthodontics and surgery [15]. The major advantages of $\mathrm{CBCT}$ in pediatric patients arise from lesser scan time and less complicated apparatus, which minimize anxiety in children. Images obtained with CBCT are highly magnified, with reduced distortion and reduced dosage. Though, CBCT has higher dose as compared with conventional radiography, the range of dose reduction is between $96 \%$ and $51 \%$ compared to conventional head CT[16].

Further, total annual effective doses from natural radiation sources to the Indian population residing in normal background areas work out to be $2.299 \mathrm{mSv} / y e a r$ [17]. It has been observed that the radiation exposure from $\mathrm{CBCT}$ is within the same range as traditional dental imaging as seen in the Table 4[18].

Moreover, a complete series of periapical radiographs has a higher radiation dose than CBCT [19]. Till date the major importance of CBCT in children has been in relation of orthodontics. However with development of newer technologies, it might become easier to diagnose caries, determine the outcome of treatment and provide real time imaging in surgical cases and also in previous studies done by Ran Yang et al showed that the root and canal morphology of primary mandibular second molars could be better visualized in CBCT than conventional radiographs [20].

Hence the use of this imaging modality should be balanced with the perspective that most CBCT scans are easier to perform in a dental office when compared with a complete series of periapical radiographs, or perhaps even a panoramic radiograph. However, use of CBCT in children should be justified based upon the case such that its application outweighs the potential hazards of radiation exposure and all the basic principles should be followed accurately [18].

\section{CONCLUSION}

The following inferences were derived from the present study:-

1. Study Apex DSP (Septodont, France) was found to be accurate in measuring the working length across a variety of irrigants commonly used in the pediatric endodontic practice for measurement of the length of the root canal in primary molar roots.

2. The contents of the root canal influenced the results of the measurements by the apex locator used with significant difference in measurement in presence of $2.5 \%$ sodium hypochlorite.

\section{Bullet points \\ What this paper adds}

The current study highlights the need for a better and easy diagnostic tool for working length determination in primary teeth. The results of the study indicate that the Apex DSP can be used efficiently for estimation of working length in presence of normal saline and chlorhexidine glucanoate as irrigating solution. 
Anindita Talukdar et al; Saudi J Oral Dent Res, Feb, 2021; 6(2): 92-96

\section{Why this paper is important for paediatric dentists}

The use of a diagnostic tool that can reduce the operating time in children and also reduce the number of radiographs to be taken.

The use of previously taken CBCT scans for reasons like malocclusion or surgery and use the scan as an adjunct when possible.

\section{ACKNOWLEDGEMENTS}

We would like to acknowledge the Department of Oral Medicine and Radiology for the armamentarium and support.

\section{REFERENCES}

1. Tosun, G., Erdemir, A., Eldeniz, A. U., Sermet, U., \& Sener, Y. (2008). Accuracy of two electronic apex locators in primary teeth with and without apical resorption: a laboratory study. International endodontic journal, 41(5), 436-441.

2. Kielbassa, A. M., Muller, U., Munz, I., \& Monting, J. S. (2003). Clinical evaluation of the measuring accuracy of ROOT ZX in primary teeth. Oral Surgery, Oral Medicine, Oral Pathology, Oral Radiology, and Endodontology, 95(1), 94-100.

3. Neena, I. E., Ananthraj, A., Praveen, P., Karthik, V., \& Rani, P. (2011). Comparison of digital radiography and apex locator with the conventional method in root length determination of primary teeth. Journal of Indian Society of Pedodontics and Preventive Dentistry, 29(4), 300.

4. Real, D. G., Davidowicz, H., Moura-Netto, C., Zenkner, C. D. L. L., Pagliarin, C. M. L., Barletta, F. B., \& de Moura, A. A. M. (2011). Accuracy of working length determination using 3 electronic apex locators and direct digital radiography. Oral Surgery, Oral Medicine, Oral Pathology, Oral Radiology, and Endodontology, 111(3), e44-e49.

5. Durack, C., Patel, S. (2012). Cone beam computed tomography in endodontics. Braz Dent J. 23,17991.

6. Fuks, A.B., Eidelman, E. (1991). Pulp therapy in the primary dentition. Current Opinion in Dentistry, 1,556-63.

7. Coll, J.A., Sadrian, R. (1996). Predicting pulpectomy success and its relationship to exfoliation and succedaneous dentition. Pediatr Dent, 18:57-63.

8. Nowak, A.J., Pediatric Dentistry. (1999). The Handbook, 2nd edn. Chicago, IL, USA, American Academy of Pediatric Dentistry.

9. Mente, J., Seidel, J., Buchalla, W., \& Koch, M. J. (2002). Electronic determination of root canal length in primary teeth with and without root resorption. International

Endodontic

Journal, 35(5), 447-452.

10. Irfana, K., Ruchika, B., Tajinder, B., Harkanwal. Preet, Singh., Madhulika, Y., \& K. Jetender, R. (2014). A comparative evaluation of working length with digital radiography and third generation apex locator (ProPex) in the presence of various intracanal irrigants: An in vivo/ex vivo study. Dent Res J, 11(1), 56-60.

11. Kobayashi, C. (1995). Electronic canal length measurement. Oral Surg Oral Med Oral Pathol Oral Radiol Endod, 79, 226-31.

12. Fan, W., Fan, B., Gutmann, JL., Bian, Z., Fan, MW. (2006). Evaluation of the accuracy of three electronic apex locators using glass tubules. Int Endod J, 39, 127-35.

13. Ozsezer, E., Inan, U., Aydin, U. (2007). In vivo evaluation of ProPex electronic apex locator. $J$ Endod., 33,974-7.

14. Lucena, C., López, J. M., Martín, J. A., Robles, V., \& González- Rodríguez, M. P. (2014). Accuracy of working length measurement: electronic apex locator versus cone- beam computed tomography. International endodontic journal, 47(3), 246-256.

15. İşman, Ö., Yılmaz, H. H., Aktan, A. M., \& Yilmaz, B. (2017). Indications for cone beam computed tomography in children and young patients in a Turkish subpopulation. International journal of paediatric dentistry, 27(3), 183-190.

16. Roberts, J. A., Drage, N. A., Davies, J., \& Thomas, D. W. (2009). Effective dose from cone beam CT examinations in dentistry. The British journal of radiology, 82(973), 35-40.

17. Ramachandran, T.V., Eappen, K.P., Nair, R.N., Mayya, Y.S., Puranik, V.D. (2005). Background radiation exposure levels: Indian scenario. Environmental Assessment Division, Bhabha Atomic Research Center, 5-6.

18. Dhillon, J. K., \& Kalra, G. (2013). Cone beam computed tomography: An innovative tool in pediatric dentistry. J Pediatr Dent, 1(2), 27-31.

19. De Vos, W., Casselman, J., \& Swennen, G. R. J. (2009). Cone-beam computerized tomography (CBCT) imaging of the oral and maxillofacial region: a systematic review of the literature. International journal of oral and maxillofacial surgery, 38(6), 609-625.

20. Yang, R., Yang, C., Liu, Y., Hu, Y., \& Zou, J. (2013). Evaluate root and canal morphology of primary mandibular second molars in Chinese individuals by using cone-beam computed tomography. Journal of the Formosan Medical Association, 112(7), 390-395. 